Original Article

\title{
Effects of antagonistic muscle contraction exercises on ankle joint range of motion
}

\author{
Katsuhito Nagano, RPT, PhD ${ }^{1)^{*}}$, SAchiyo Uoya, RPT ${ }^{2)}$, Yumi Nagano, RPT ${ }^{3)}$ \\ 1) Division of Physical Therapy, Department of Rehabilitation, Faculty of Health Science, \\ Fukui Health Science University: 56 Egami-cho 13-1, Fukui city, Fukui 910-3190, Japan \\ 2) Department of Rehabilitation, Ohmori Clinic for Orthopaedics and Rheumatology, Japan \\ 3) Department of Nursing Care, Medical Care Co., Ltd., Japan
}

\begin{abstract}
Purpose] Antagonistic stretching is an important therapeutic program in rehabilitation. However, if pain occurs during stretching, other methods should be considered. This study aimed to investigate whether antagonistic muscle contraction exercises alone had an effect in expanding the joint range of motion. [Participants and Methods] Participants included 50 healthy males and females (aged 21.0 \pm 3.1 years) who were each randomly assigned to one of four groups that performed either active, resistance, phasic, or isometric exercises or a control group that performed no exercise. Each exercise group performed five sets of 30-second ankle dorsiflexion exercises. Active and passive ranges of motion of ankle dorsiflexion were measured using a goniometer, with the participants in the knee extension and flexion positions. [Results] Ankle joint range of motion was increased in the knee extension position in the isometric exercise group. There were no other differences observed in any of the groups. [Conclusion] Our results indicated that isometric exercises had an immediate effect on increasing ankle joint range of motion. This is presumably attributed to the effects of Golgi tendon organ inhibition (Ib inhibition) or stretch tolerance of the gastrocnemius muscle.

Key words: Isometric exercise, Antagonistic muscle contraction, Joint range of motion
\end{abstract}

(This article was submitted Feb. 14, 2019, and was accepted Apr. 3, 2019)

\section{INTRODUCTION}

Limited range of motion (ROM) occurs due to trauma, disease, and immobility by cast or splint and can lower daily living activities or sports performance ${ }^{1)}$. The ankle plantar-flexor muscles are involved in maintaining the standing position or balance and are known as antigravity muscles. In addition, they affect mobility in dorsiflexion exercise via relaxation ${ }^{2)}$. Fixation with a cast to treat ankle sprains or tarsal bone fractures and consequent exercise of the ankle joint can decrease the extension of the plantar muscle and surrounding tissues and limit the ankle dorsiflexion $\mathrm{ROM}^{3}$. A sufficient ankle dorsiflexion angle is required for squatting or for rising from the floor or a chair, and ankle dorsiflexion restrictions lower toe clearance during walking, going up and down the stairs, and striding; further, the ankle dorsiflexion angle is reportedly a factor of stumbling, which leads to falls ${ }^{4,5)}$.

Methods to increase joint ROM include joint ROM exercises, in which the joints are actively or passively moved to the maximum ROM; stretching, in which the antagonistic muscles and tendon tissues are extended; agonist muscle strength training exercise therapy; continuous passive motion; and use of robots ${ }^{3,6}$. Of these, stretching is versatile in clinical practice. Stretching includes static or ballistic stretching and proprioceptive neuromuscular facilitation (PNF), and all these methods are known to effectively and immediately increase the ROM. Unlike simple stretching, the hold-relax PNF method combines static stretching and isometric contraction exercises of the muscle to be extended, and it is reportedly the most effective

*Corresponding author. Katsuhito Nagano (E-mail: nagano@xg8.so-net.ne.jp)

(C2019 The Society of Physical Therapy Science. Published by IPEC Inc.

(c) (1) $($ This is an open-access article distributed under the terms of the Creative Commons Attribution Non-Commercial No Derivatives

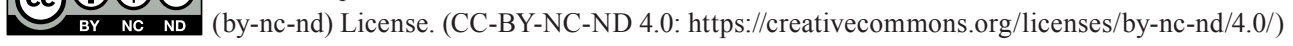


among all methods ${ }^{7}$. Immediate joint ROM increases are reportedly achieved in PNF stretching by performing isometric contraction exercises at maximum or submaximum contraction, which is $80 \%$ of the maximum contraction, combined with stretching lasting for $\geq 30 \mathrm{~s} /$ bout for a total of $\geq 1 \mathrm{~min}^{8,9)}$. Such methods to increase joint ROM involve stretching antagonist muscles, with the exception of muscle strength training, and there are few methods with which to increase joint ROM without stretching. Limited joint ROM may be accompanied by pain on extension in the antagonist muscles; thus, a method to improve joint ROM without stretching antagonist muscles is needed.

However, the effects of joint ROM increase caused by antagonist muscle contraction exercise without stretching or the differences between the types of muscle contraction exercises are unknown. Thus, this study aimed to investigate the immediate effects of isotonic contraction and isometric contraction as well as resistance and phasic exercises without stretching of the ankle plantar-flexor muscles on ankle dorsiflexion ROM.

\section{PARTICIPANTS AND METHODS}

The study included healthy individuals ( 25 males and 25 females; age, $21.0 \pm 3.1$ years) and was approved by the ethics review committee of Nittazuka Medical Welfare Center (approval no. 29-111). Furthermore, the nature and details of the study were explained to all participants and written informed consent was obtained prior to initiating the study. The characteristics of participants in the study are shown in Table 1.

The participants were randomly assigned to one of the following five groups: active, phasic, resistance, or isometric exercise groups or the control group (10 participants/group) using the Excel computer program (version 2013; Microsoft Corporation, Redmond, WA, USA).

The control group was assessed in the resting supine position without performing any exercise. Using a metronome, the active exercise group performed the ankle plantar-flexion exercise in the supine position at a rate of 1 bout/s from the central to maximal plantar-flexion position. The phasic exercise group also performed the ankle plantar-flexion exercise from the central to maximal plantar-flexion position at a rate of 2 bouts/s using a metronome. The resistance exercise group performed the ankle plantar exercise from the central to maximal plantar-flexion position in the supine position at a rate of 1 bout/s using a TheraBand ${ }^{\circledR}$ (The Hygenic Corp., Akron, OH, USA) and a metronome. The strength of the TheraBand ${ }^{\circledR}$ was adjusted to a length at which the participant could expand the TheraBand ${ }^{\circledR}$ to its limit. Participants were verbally instructed to utilize their maximum strength when performing plantar-flexion exercises. Finally, the isometric exercise group was made to sit in the pelvic retroversion long-sitting position to prevent any effect on the hamstring. To prevent the body from shifting backwards due to a plantar-flexion reaction, the arms were placed diagonally backwards with the soles of the feet in contact with the wall while exerting a sustained push against the same. During this time, participants were verbally instructed to keep pushing against the wall with maximum strength. All exercises were performed in 5 sets of $30 \mathrm{~s}$ each, with a 2.5 -min interval between each set. The ankle-dorsiflexion angle was measured according to the measurement method of Tazawa et al. ${ }^{10)}$ and Kumamoto et al ${ }^{11)}$. Briefly, the line connecting the head of the fibula and lateral malleolus as the base axis and that between the base and bone head of the fifth metatarsal with the metatarsal head as the axis of motion was measured, and thin lines were drawn on the skin using a marker pen. Examiner 1 manually fixed the knee joint and lower leg to prevent compensation in the lower leg, Examiner 2 fixed the ankle dorsiflexion's final range of motion, Examiner 3 set the goniometer to each axis, Examiner 4 confirmed that the goniometer and axes were properly aligned, and Examiners 3 and 4 simultaneously read the goniometer scale positions twice. During measurement of the ankle joint dorsiflexion angle, the active joint movement range and passive joint movement range in the knee extension positions and knee flexion positions were also measured.

Measurements are expressed as mean \pm standard deviation. The statistical analysis software Statcel4 (OMS Ltd.) was used to assess normality, and the paired t-test was used to compare post-intervention measurements with baseline. Significance levels of $<5 \%$ were considered statistically significant.

\section{RESULTS}

The ankle-dorsiflexion angle was compared before and after intervention in the control, active, phasic, and isometric exercise groups. No significant differences were observed between baseline and post-intervention values of passive and active ROM in the knee flexion position and passive ROM in the knee extension position of the active, phasic, and isometric exercise groups and the control group. However, the active ROM in the knee extension position significantly increased in the isometric exercise group after intervention compared with that before the intervention (Table 2).

\section{DISCUSSION}

This study investigated whether active, phasic, resistance, or isometric exercise increased ankle ROM without stretching, 
Table 2. The rate of ROM increase in pre and post exercise (\%)

\begin{tabular}{lccccc}
\hline \multirow{2}{*}{ Measurement method } & \multicolumn{5}{c}{ Group } \\
\cline { 2 - 6 } & Control & Active & Phasic & Resistance & Isometric \\
\hline Active ROM in knee flexion position & $106.4 \pm 23.5$ & $100.7 \pm 26.6$ & $103.0 \pm 32.5$ & $105.1 \pm 24.0$ & $116.6 \pm 31.4$ \\
Passive ROM in knee flexion position & $94.2 \pm 11.2$ & $98.5 \pm 9.2$ & $95.9 \pm 13.0$ & $102.1 \pm 10.5$ & $94.2 \pm 9.6$ \\
Active ROM in knee extention position & $101.2 \pm 25.6$ & $88.2 \pm 33.0$ & $97.5 \pm 27.0$ & $116.6 \pm 29.6$ & $119.9 \pm 22.5^{* *}$ \\
Passive ROM in knee extention position & $100.1 \pm 15.3$ & $110.7 \pm 15.2$ & $91.7 \pm 13.1$ & $102 \pm 13.3$ & $107.5 \pm 11.4$ \\
\hline
\end{tabular}

Data are expressed as mean \pm SD. ROM: range of motion.

**Significant $(\mathrm{p}<0.01)$ difference between pre and post exercise within group.

Control: no exsecise group. Active: active exercise group. Phasic: phasic exercise group. Resistance: resistance exercise group.

Isometric: isometric exercise group.

which is considered essential for increasing joint ROM. Results of a comparison between post-intervention and baseline measurements demonstrated that isometric exercise increased ankle dorsiflexion ROM with the knee in the flexion position. In contrast, active, phasic, and resistance exercise were found to have little effect on increasing ankle ROM.

Golgi tendon organ inhibition ( $\mathrm{Ib}$ inhibition) or decreased tension caused by decreased excitation of $\gamma$ motor neurons of antagonistic muscles, decreased musculotendinous stiffness, changes in musculotendinous structures, muscle fatigue, increased pennation angle, muscle temperature, and stretch resistance are all reportedly associated with ankle ROM ${ }^{12)}$.

In general, the physiological action of Ib inhibition involves tension to tendons that excites the Golgi tendon organ (GTO), which inhibits the excitation of $\gamma$ motor neurons to ultimately decrease muscle tension ${ }^{13,14)}$. Isometric exercise requires maximum muscle strength and involves sustained muscle contraction at a constant joint angle. Accordingly, this muscle contraction force presumably increases tendinous tension to excite the GTO to consequently induce Ib inhibition.

Therefore, the increased angle ROM by isometric contraction exercise presented in this study is assumedly one of the factors that explain how sustained muscle contraction induces Ib inhibition, which decreases muscle tension to increase muscle extensibility. However, Ib inhibition is unlikely to occur specifically during isometric contraction, because tendinous tension is expected to increase during muscle contraction in exercises other than isometric exercise. Bandy et al. ${ }^{15)}$ reported no significant difference between the effects of 30 - and 60-s static stretches on the flexibility of the hamstring muscles. However, they reported that compared to these two durations, a 15-s stretch was less effective. In the present study, a high, sustained tension was exerted on the tendon for $30 \mathrm{~s}$ in the isometric contraction group, whereas a high tendinous tension occurred only during contraction of the contraction-relaxation cycle in the other three groups that performed isotonic contraction exercises, thereby indicating that the duration of the effects of tendinous tension was possibly shorter than in isometric contraction. This temporal difference is possibly one of the factors that lowered the Ib inhibition effect on the inhibitory mechanisms of muscle tension, which ultimately resulted in no joint ROM increase in the groups other than the isometric contraction group.

Regarding the influence of musculotendinous stiffness, the relationship between the force that moves the joint and the length of muscle or tendon extension has been represented by a linear regression line, and the term "stiffness" has been used to define the resistance that occurs when a muscle or tendon is longitudinally extended ${ }^{16,17)}$. Konrad et al. compared the effects of static, ballistic, and PNF stretching on the triceps surae and reported that stretching decreased muscle stiffness but not tendon stiffness ${ }^{18)}$. In the present study, increases in muscle contraction and tendinous tension occurred in all exercise groups. However, the muscles never reached the maximum extension position even if the tendon did, because the muscles and tendons were not directly stretched. The exercises performed in each group had little effect on muscle stiffness owing to the low direct extension effects on the muscle and the fact that tendon stiffness did not change despite an extension force being exerted on the tendon, thus highlighting a slight relationship between increased joint ROM and musculotendinous stiffness.

Fatigue from exercise reportedly decreases joint $\mathrm{ROM}^{19}$. However, in the present study, post-exercise ROM did not decrease for any of the exercise groups, thereby indicating that the total amount of exercise performed by each group did not cause enough fatigue to decrease ROM.

A factor additional to those mentioned above has been described by Magnusson et $\mathrm{a}^{20)}$. They applied passive stretching to the hamstring muscles and reported that joint ROM increased by this stretching was attributed to increased stretch tolerance and not to a change in mechanical or viscoelastic properties of the muscle. Furthermore, Mizuno ${ }^{21)}$ reported that ROM increased without changing the mechanical properties of the muscle-tendon unit in a group that performed $\geq 4$ sets of dynamic stretching, thus indicating that the ROM increased by isometric contraction exercise in the present study was due to increased stretch tolerance and not musculotendinous structural changes. Therefore, isometric contraction exercise on antagonist muscles instantaneously increased the ROM, and increased ROM was presumed to be associated with the duration of muscle contraction and intensity of musculotendinous tension. Furthermore, this study suggested that increased ROM was not associated with changes to musculotendinous structures but was a result of Ib inhibition or stretch tolerance.

\section{Conflict of interest}

The authors declare no conflicts of interest. 


\section{REFERENCES}

1) Hemmerich A, Brown H, Smith S, et al.: Hip, knee, and ankle kinematics of high range of motion activities of daily living. J Orthop Res, 2006, 24: 770-781. [Medline] [CrossRef]

2) Caplan N, Forbes A, Radha S, et al.: Effects of 1 week of unilateral ankle immobilization on plantar-flexor strength, balance, and walking speed: a pilot study in asymptomatic volunteers. J Sport Rehabil, 2015, 24: 156-162. [Medline] [CrossRef]

3) Zhang M, Davies TC, Zhang Y, et al.: Reviewing effectiveness of ankle assessment techniques for use in robot-assisted therapy. J Rehabil Res Dev, 2014, 51: 517-534. [Medline] [CrossRef]

4) Dill KE, Begalle RL, Frank BS, et al.: Altered knee and ankle kinematics during squatting in those with limited weight-bearing-lunge ankle-dorsiflexion range of motion. J Athl Train, 2014, 49: 723-732. [Medline] [CrossRef]

5) Rosenblatt NJ, Bauer A, Rotter D, et al. CPO: Active dorsiflexing prostheses may reduce trip-related fall risk in people with transtibial amputation. J Rehabil Res Dev, 2014, 51: 1229-1242. [Medline] [CrossRef]

6) Harvey LA, Brosseau L, Herbert RD: Continuous passive motion following total knee arthroplasty in people with arthritis. Cochrane Database Syst Rev, 2014, 6: CD004260 10.1002/14651858.CD004260.pub3. [Medline]

7) Wicke J, Gainey K, Figueroa M: A comparison of self-administered proprioceptive neuromuscular facilitation to static stretching on range of motion and flexibility. J Strength Cond Res, 2014, 28: 168-172. [Medline] [CrossRef]

8) Behm DG, Blazevich AJ, Kay AD, et al.: Acute effects of muscle stretching on physical performance, range of motion, and injury incidence in healthy active individuals: a systematic review. Appl Physiol Nutr Metab, 2016, 41: 1-11. [Medline] [CrossRef]

9) Davis DS, Ashby PE, McCale KL, et al.: The effectiveness of 3 stretching techniques on hamstring flexibility using consistent stretching parameters. J Strength Cond Res, 2005, 19: 27-32. [Medline]

10) Tazawa R, Hasegawa M, Onuma R, et al.: Reproducibility of reading angle differences during range of motion tests. Bull Ryotokuji Univ, 2013 , 7: 83-88.

11) Kumamoto T, Kobayashi T, Kubota K: Reliability of measuring range of motion among students. Hokkaido J Phys Ther, 2010, 27: 18-20.

12) Konrad A, Gad M, Tilp M: Effect of PNF stretching training on the properties of human muscle and tendon structures. Scand J Med Sci Sports, 2015, 25: 346-355. [Medline] [CrossRef]

13) Chalmers G: Re-examination of the possible role of Golgi tendon organ and muscle spindle reflexes in proprioceptive neuromuscular facilitation muscle stretching. Sports Biomech, 2004, 3: 159-183. [Medline] [CrossRef]

14) Miller KC, Burne JA: Golgi tendon organ reflex inhibition following manually applied acute static stretching. J Sports Sci, 2014, 32: 1491-1497. [Medline]

15) Bandy WD, Irion JM: The effect of time on static stretch on the flexibility of the hamstring muscles. Phys Ther, 1994, 74: 845-850, discussion 850-852. [Medline] [CrossRef]

16) McMahon G, Morse CI, Winwood K, et al.: Gender associated muscle-tendon adaptations to resistance training. PLoS One, 2018, 13: e0197852 [CrossRef]. [Medline]

17) Morgan DL, Proske U, Warren D: Measurements of muscle stiffness and the mechanism of elastic storage of energy in hopping kangaroos. J Physiol, 1978, 282: 253-261. [Medline] [CrossRef]

18) Konrad A, Stafilidis S, Tilp M: Effects of acute static, ballistic, and PNF stretching exercise on the muscle and tendon tissue properties. Scand J Med Sci Sports, 2017, 27: 1070-1080. [Medline] [CrossRef]

19) Dashottar A, Costantini O, Borstad J: A comparison of range of motion change across four posterior shoulder tightness measurements after external rotator fatigue. Int J Sports Phys Ther, 2014, 9: 498-508. [Medline]

20) Magnusson SP, Simonsen EB, Aagaard P, et al.: A mechanism for altered flexibility in human skeletal muscle. J Physiol, 1996, 497: 291-298. [Medline] [CrossRef]

21) Mizuno T: Changes in joint range of motion and muscle-tendon unit stiffness after varying amounts of dynamic stretching. J Sports Sci, $2017,35: 2157-2163$. [Medline] [CrossRef] 